\title{
Commentary on the SAGES Guidelines for Surgical Treatment of Gastroesophageal Reflux Disease
}

\author{
Vic Velanovich
}

Published online: 28 July 2010

(C) Springer Science+Business Media, LLC 2010

The SAGES Guidelines Committee has created a document on the surgical management of gastroesophageal reflux disease (GERD) using the most rigorous methods of systematic review. From this standpoint, the information provided is of the highest quality and the recommendations rest on a solid foundation. All surgeons who care for patients with GERD can look to this document for guidance and support. As reflux disease continues to be a common malady, with well over US $\$ 1$ billion spent on direct costs, and with an unknown but undoubtedly large amount spent on indirect costs, this is a topic worthy of comparative clinical effectiveness analysis.

It must be remembered, however, that these Guidelines are written for the surgical management of GERD. Appropriate references are made to guidelines dealing with medical management, and an analysis of the extant controlled trials comparing medical with surgical management is presented. However, the Guidelines do not settle the controversy regarding whether medical or surgical therapy is superior. This is appropriate, since GERD is a protean disease in its patients and manifestations. No single treatment plan is appropriate for all patients.

The indications for an operation are based specifically on the pathology of gastroesophageal reflux, not on the presence of a hiatal hernia. Therefore, the indications for an operation for a paraesophageal hernia are not a part of these Guidelines. However, the indications for an operation for GERD presented are clear, circumscribed, and comprehensive.

The preoperative evaluation of the patient with GERD has been an area of confusion-which tests are needed and for

V. Velanovich $(\square)$

Division of General Surgery, K-8, Henry Ford Hospital,

2700 West Grand Blvd., Detroit, MI 48202, USA

e-mail: vvelano1@hfhs.org whom? These Guidelines provide an evidence-based answer: enough testing to confirm pathologic reflux. Upper gastrointestinal endoscopy is mandatory to confirm mucosal injury as a result of acid reflux. This author believes that endoscopy is also important to rule out other, more sinister, pathologies such as Barrett's esophagus or even adenocarcinoma. If no such injury is visualized, then 24-h esophageal $\mathrm{pH}$ monitoring is mandatory to confirm pathologic reflux. Interestingly, the authors did not find evidence supporting esophageal manometry and gastric emptying scintigraphy. In my opinion, manometry is important to rule out other esophageal motility disorders which may preclude an antireflux operation. As preoperative bloating in not uncommonly found in GERD patients, gastric emptying scintigraphy may identify patients with coexisting gastroparesis in whom concomitant pyloroplasty may be of benefit.

In understanding the results of the studies on the comparative treatments for GERD, one must clearly keep in mind the goals of treatment. Much of the controversy regarding medical versus surgical management relates to which endpoints are emphasized: Symptomatic relief, reduction of esophageal acid exposure, reduction in medication use, patient satisfaction, and costs have all been applied as primary endpoints. These endpoints measure different, albeit related, things. The only patient-centered outcomes are symptomatic relief and satisfaction; however, these symptoms, whether occurring preoperatively or postoperatively, may not, in fact, be related to acid reflux. Reduction of acid exposure is the only physiologically objective endpoint, but many of the symptoms of GERD are related to reflux volume, such as regurgitation, or bile reflux, independent of the acid content of the refluxate. The resumption of the use of medications, primarily proton-pump inhibitors (PPIs), is almost always based on a physician or patient judging that postoperative symptoms are due to a failure of the operation rather 
than a comprehensive search for the true cause of the symptoms. Postoperative symptoms may be related to persistent or recurrent acid reflux (a true failure of the operation), or to other causes, such as functional esophageal disorders. Therefore, many patients who are not refluxing acidic gastric contents are placed on postoperative PPIs, causing a delay in the diagnosis of the true cause of their symptoms. The importance of costs depends upon the stakeholder's viewpoint: the greater society's, the patient's, the physician's, the payer's, or the hospital's. These viewpoints may be in conflict with one another. Although, in an egalitarian world, the greater society's view would be the most important, decisions by less comprehensive stakeholders rarely take this viewpoint into account.

Another area of controversy for which the Guidelines provide solid information has to do with the salient steps in performing an antireflux operation. Issues such as the comparative effectiveness of an open versus laparoscopic approach, and complete versus partial fundoplication, are addressed and settled based upon solid level I evidence. The laparoscopic approach is clearly preferred, when it can be done safely. Either partial or complete fundoplication is acceptable as long as the surgeon constructs the fundoplication well, and this is the important point: $\mathrm{We}$, as surgeons or other medical professionals, have a tendency to blame the operation when we should blame how the operation was done. A good operation done badly will lead to poor results.

Additional controversial technical considerations are addressed, such as division of the short gastric vessels. Here, despite level I evidence suggesting that division of the short gastric vessels is unnecessary, the authors yield to "expert opinion in North America" that the short gastric vessels should be divided. Clearly, the controversy here continues. Other areas of controversy include robotic surgery, Roux-en-Y gastric bypass as an alternative to fundoplication in the morbidly obese, and use of dilators. These, I believe, have been satisfactorily addressed.

Nontechnical factors which may affect symptomatic outcomes have also been addressed. These include the presence of depression, anxiety, and other psychological issues. These are areas in which surgeons need to have a clear understanding. The Guidelines provide an excellent review of outcomes, with a good balance of symptomatic outcomes and adverse events. This information is of particular value for the surgeon in patient counseling.

There are areas that these Guidelines do not address. Endoscopic versus endoluminal approaches to GERD have not been addressed, although the authors refer to a recent review published in Surgical Endoscopy. Nevertheless, if a surgeon is considering any endoluminal approach, these guidelines will not be helpful.

In summary, these Guidelines are noteworthy for the comprehensiveness of the data review as well as their succinctness. After each data review, clear recommendations are given. As with any systematic review, analyzing the best practices available from the standpoint of level of evidence is not the same as interpreting data for an individual patient-related decision, as the data may or may not be applicable to any one individual patient. This still is the job of the treating surgeon.

Disclosures The author Vic Velanovich has no conflicts of interest or financial ties to disclose. 